\title{
Traveling Waves Solutions for Delayed Temporally Discrete Non-Local Reaction-Diffusion Equation
}

\author{
Hongpeng Guo and Zhiming Guo *
}

check for

updates

Citation: Guo, H.; Guo, Z. Traveling Waves Solutions for Delayed Temporally Discrete Non-Local Reaction-Diffusion Equation. Mathematics 2021, 9, 1999. https:/ / doi.org/10.3390/math9161999

Academic Editor: Ravi P. Agarwal

Received: 9 July 2021

Accepted: 18 August 2021

Published: 20 August 2021

Publisher's Note: MDPI stays neutral with regard to jurisdictional claims in published maps and institutional affiliations.

Copyright: (c) 2021 by the authors. Licensee MDPI, Basel, Switzerland. This article is an open access article distributed under the terms and conditions of the Creative Commons Attribution (CC BY) license (https:/ / creativecommons.org/licenses/by/ $4.0 /)$.
School of Mathematics and Information Science, Guangzhou University, Guangzhou 510006, China; guohongpeng8013@126.com

* Correspondence: guozm@gzhu.edu.cn

\begin{abstract}
This paper deals with the existence of traveling wave solutions to a delayed temporally discrete non-local reaction diffusion equation model, which has been derived recently for a single species with age structure. When the birth function satisfies monotonic condition, we obtained the traveling wavefront by using upper and lower solution methods together with monotonic iteration techniques. Otherwise, without the monotonicity assumption for birth function, we constructed two auxiliary equations. By means of the traveling wavefronts of the auxiliary equations, using the Schauder' fixed point theorem, we proved the existence of a traveling wave solution to the equation under consideration with speed $c>c^{*}$, where $c^{*}>0$ is some constant. We found that the delayed temporally discrete non-local reaction diffusion equation possesses the dynamical consistency with its time continuous counterpart at least in the sense of the existence of traveling wave solutions.
\end{abstract}

Keywords: temporally discrete; reaction-diffusion equation; traveling wave solutions; upper-lower solution; Schauder's fixed point; delay

\section{Introduction}

Very recently, we derived in [1] the following delayed temporally discrete non-local reaction-diffusion equation

$$
w(n+1, x)=D \Delta_{x} w(n+1, x)+(1-d) w(n, x)+\eta \int_{-\infty}^{+\infty} K_{D_{i}}(r-1, x-y) b(w(n+1-r, y)) d y,
$$

where $w(n, x)$ denotes the density of the total matured population of a single species at time $n \in \mathbb{N}$, location $x \in \mathbb{R}, \mathbb{N}$ represents the set of non-negative integers. $D>0$ is the diffusion rate of matured individuals. $\Delta_{x}$ is the Laplacian operator with respect to $x \in \mathbb{R}$, $r \geq 2$ is a positive integer, which denotes the maturation time for the species. $0<d<1$ represents the death rate of matured individuals. $0<\eta<1$ is the survival rate of an individual from birth to maturation. $b(\cdot)$ is the birth function. The integral kernel function $K_{D_{i}}$ reads as

$$
K_{D_{i}}(r-1, x)=\frac{1}{\sqrt{D_{i}}} e^{-\frac{|x|}{\sqrt{D_{i}}}} \sum_{m=0}^{r-2} \frac{(r-1) \cdot r \cdots(r+m-2)}{m !(r-2-m) ! 2^{r+m-1}}\left(\frac{|x|}{\sqrt{D_{i}}}\right)^{r-2-m},
$$

where $D_{i}$ is the diffusion rate of immatured individuals. In particular, we use the convention that if $m=0$ then $m !=1$ and $(r-1) r \cdots(r+m-2)=1$.

For the case $r=1, K_{D_{i}}(r-1, x)=K_{D_{i}}(0, x)=\delta(x)$, i.e., the Dulac $\delta$ function. Therefore, (1) is reduced to

$$
w(n+1, x)=D \Delta_{x} w(n+1, x)+(1-d) w(n, x)+\eta b(w(n, x)),
$$


Equation (1) reflects the changes of matured population of a single species in an unbounded habitat with dimension one. It can also be viewed as a non-standard discretization of the following well-known non-local reaction-diffusion equation model with delay,

$$
\frac{\partial w}{\partial t}=D \frac{\partial^{2} w}{\partial x^{2}}-d w+\varepsilon \int_{-\infty}^{\infty} b(w(t-r, y)) f_{\alpha}(x-y) d y,
$$

which was derived in 2001 by So, Wu and Zou [2]. The model (4) describes the adult population's evolution of a single species that have two age classes in an unbounded spatial domain $\mathbb{R}^{1}$. In above Equation (4), $D>0$ is the diffusion rate of the adult population and $d>0$ is the death rate of the adult population. $\varepsilon>0$ reflects the impact of the death rate of the immature on the matured population. $b(\cdot)$ is the birth function. $r>0$ is the maturation time of the species. $\alpha \geq 0$ also reflect the impact of the dispersal rate of the immature on the matured population. The integral kernel function is $f_{\alpha}(x)=\frac{1}{\sqrt{4 \pi \alpha}} e^{\frac{-x^{2}}{4 \alpha}}$. When $\alpha$ approaches to 0 , that is, the immature do not disperse, (4) is reduced to

$$
\frac{\partial w}{\partial t}=D \frac{\partial^{2} w}{\partial x^{2}}-d w+\varepsilon b(w(t-r, x))
$$

and the non-local effect disappears.

In our previous paper [1], we have already given a detailed derivation of Equation (1). Especially, when $D_{i}=0$ (the immature individuals do not disperse), (1) becomes

$$
w(n+1, x)=D \Delta_{x} w(n+1, x)+(1-d) w(n, x)+\eta b(w(n+1-r, x)) .
$$

Above equation is a non-standard temporally discrete version of (5).

It is well known that the continuous-time reaction-diffusion equation has been widely used to describe diffusive phenomena in physics, engineering, chemistry, biology, and so on (see, for example, [3-10]). In general, the dynamical behaviors of solutions to a nonlinear reaction-diffusion equation are very complicated, and it is often very difficult to find exact solutions. For the sake of understanding the properties of the solutions numerically, we need to study its discrete analogue. However, the basic principle of constructing appropriate discretization of differential equations is to preserve the properties of the corresponding differential equations. Since many classical (standard) discretizations cannot achieve dynamic consistency, non-standard discretizations are usually used to ensure it. Thus, Mickens first introduced the concept of dynamical consistency in [11,12] for ordinary differential equations and since then, some dynamical consistent discrete schemes have been constructed. See, for example, Refs. [13-17], and references therein.

In [1], we established the existence of traveling wavefronts of Equation (6) by using upper and lower solution methods and iterate techniques. We found that (6) possesses the dynamical consistency with its time continuous counterpart (5) at least in the sense of the existence of traveling wave solutions. In the sense of propagation, Equation (6) is also a good approximation of corresponding continuous time model (5).

From the perspective of mathematical biological modeling, almost all of the data collected are discrete in time because observations are always discontinuous. For example, satellite photographs used for scientific research are usually taken periodically, but the spatial distribution can be seen as continuous. Temporally discrete and spatially continuous diffusion model will be more suitable than its corresponding time continuous diffusion model to study the dynamic behavior of a single species that living in a spatially continuous habitat in population ecology. In 2002, Weinberg, Lewis, and Li in [18] gave some reasons for studying discrete-time models rather than just reaction diffusion models. They also pointed out the advantage of a discrete-time model over a reaction-diffusion model. We note that in [18], the authors studied the discrete-time recursion system

$$
u_{n+1}=Q\left[u_{n}\right],
$$


where $u_{n}(x)$ denotes the population distributions of species and $Q$ is an operator that models the growth, migration, and interaction of the species.

Although Equations (1) and (6) are different from (7) formally, we have proved in [1] that the following general non-linear equation

$$
w(n+1, x)=D \Delta_{x} w(n+1, x)+(1-d) w(n, x)+f(n, w(n, x)), x \in \mathbb{R} .
$$

is equivalent to an integral-difference equation as below,

$$
w(n+1, x)=\int_{-\infty}^{+\infty} k(x-y)[(1-d) w(n, y)+f(n, w(n, y))] d y,
$$

where $k(x)=\frac{1}{2 \sqrt{D}} e^{-\frac{|x|}{\sqrt{D}}}$. Clearly, (9) is a special case of (7) with

$$
Q(w(n, x))=\int_{-\infty}^{+\infty} k(x-y)[(1-d) w(n, y)+f(n, w(n, y))] d y .
$$

As for the general integro-difference equations,

$$
u_{n+1}(x)=\int_{\Omega} k(n, x, y) f\left(n, u_{n}(y)\right) d y,
$$

Kot and Schaffer [19] are the first to apply it modeling temporally discrete and spatially continuous dispersal phenomena, and studying the dispersal of a single species with nonoverlapping generations. They showed that, the above model will has more complex dynamic behavior than its corresponding time-continuous one. Moreover, even chaos could occur.

Since the selection of kernel function $k(n, x, y)$ plays a key role in the dynamical behavior of (10), using such a model to describe some biological phenomena will have some uncertainty. Especially, when we discuss dynamical behaviors of populations of some species living in a bounded domain, the choice of suitable integral kernels is very difficult because we may cope with various different boundary value problems.

In contrast with integral difference equations, we found that there is no such problems for temporally discrete reaction diffusion equations like (8) (or (1)). Furthermore, from the point of view of the mathematical modeling, (8) (or (1)) has the same biological explanations as those for integral difference equations. In fact, in our previous paper [1], the life cycle of individuals of the population is divided into relatively sedentary and dispersal stages. This coincides with the explanations by Kot and Schaffer [19] in establishing Equation (7). To distinguish the difference of dynamical behaviors which occurs at different stages, we assume that the evolution (the relatively sedentary stage) occurs at time $n$ and dispersal occurs at time $n+1$.

For temporally discrete reaction diffusion models, there are only a few results in the literature. In 2006, Lin and Li [20] studied following equation with delay:

$$
u_{n}(x)-u_{n-1}(x)=d \Delta u_{n}(x)+f\left(u_{n}(x), u_{n-\tau}(x)\right), n \in \mathbb{N}, x \in \mathbb{R} .
$$

They established the existence of traveling wavefronts and showed that (11) is a good approximation of its continuous time model in the sense of propagation. For more researches on this topic see $[4,21,22]$. However, we note that in the existing research literature, researchers simply assumed that the non-standard discretizations preserve the dynamical consistency of the continuous-time reaction-diffusion equations, but they do not provide reasonable biological explanations for the modeling process.

Although (1) has been derived in [1], the existence of traveling wave solutions is proved without non-local effect, and monotonic condition for birth function is assumed. In order to better understand the dynamical behaviors of (1) with non-local diffusion caused 
by immature individuals dispersion, we will study traveling wave solutions whenever the birth function is monotonic or non-monotonic, respectively.

The rest of this paper is organized as follow. In Section 2, by using the upper-lower solutions and monotone iteration technique, we studied the existence of traveling wavefronts of (1) for the cases that the birth function $b(w)$ is increasing in $\left[0, w^{*}\right]$, where $w^{*}>0$ is the unique solution to the equation $\eta b(w)=d w$. As for the case that birth function $b(w)$ is non-monotone in $\left[0, w^{*}\right]$, the theory of monotone dynamical system cannot be directly used. By using a similar idea as Ma in [23,24], we establish the existence of traveling waves of (1) in Section 3. Finally, we give a short discussion in Section 4.

\section{Traveling Wavefronts for the Monotone Case}

In this section, we will consider the existence of traveling wavefronts to equation (1) for monotone case. We are interested in finding traveling waves $w(n, x)=\varphi(x+c n)$ of following equation

$$
w(n+1, x)=D \Delta w(n+1, x)+(1-d) w(n, x)+\eta \int_{-\infty}^{+\infty} K_{D_{i}}(r-1, x-y) b(w(n+1-r, y)) d y .
$$

For this purpose, we will find a solution $\varphi(\xi)$ to (12) with $\xi=x+c n$. Clearly, $\varphi(\xi)$ satisfies the following wave profile equation

$$
\varphi(\xi+c)=D \varphi^{\prime \prime}(\xi+c)+(1-d) \varphi(\xi)+\eta \int_{-\infty}^{+\infty} b(\varphi(\xi+c-y-c r)) K_{D_{i}}(r-1, y) d y .
$$

Let $\xi^{\prime}=\xi+c$ and still denote it by $\xi$. Then, the above equation becomes

$$
-D \varphi^{\prime \prime}(\xi)+\varphi(\xi)=(1-d) \varphi(\xi-c)+\eta \int_{-\infty}^{+\infty} b(\varphi(\xi-y-c r)) K_{D_{i}}(r-1, y) d y
$$

Throughout this section, we always assume that

Hypothesis 1 (H1). $b(w)=w g(w)$, where $g(w)$ is a continuously differentiable function and satisfying $g(w)>0, g^{\prime}(w)<0$ for $w \geq 0$ and $\lim _{w \rightarrow \infty} g(w) \rightarrow 0$;

Hypothesis 2 (H2). $b(w)$ and $b^{\prime}(w)$ are bounded;

Hypothesis 3 (H3). $d<b^{\prime}(0) \eta=g(0) \eta$.

From (H1) and (H3), Equation (12) has only two constant equilibria $w=0$ and $w=w^{*}$, where $w^{*}$ is the unique solution of the equation $\eta g(w)=d$.

We will study the existence of non-decreasing solutions to Equation (14) subject to the boundary value conditions

$$
\lim _{\xi \rightarrow-\infty} \varphi(\xi)=0, \lim _{\xi \rightarrow+\infty} \varphi(\xi)=w^{*} .
$$

Our approach is similar to that in [1], which is based on the monotonic iteration techniques combined with upper and lower solution methods that was developed in [2]. To this end, we further assume that

Hypothesis 4 (H4). $b(w)$ is increasing in $\left[0, w^{*}\right]$.

To proceed further, for readers' convenience, we introduce some results on the following temporally discrete reaction-diffusion equation which will be used later,

$$
u(n+1, x)-u(n, x)=D \Delta_{x} u(n+1, x)+f(u(n, x)),
$$


where $f(\cdot)$ is assumed to be a continuous function. For detailed proofs on these results, readers can refer to [1].

Lemma 1 ([1]). The initial value problem

$$
\left\{\begin{array}{l}
u(n+1, x)-u(n, x)=D \Delta_{x} u(n+1, x), x \in \mathbb{R}, n \geq 0 . \\
u(0, x)=\varphi(x)
\end{array}\right.
$$

has a unique solution $u(n, x)$ and

$$
u(n, x)=\int_{-\infty}^{\infty} K(n, x-y) \varphi(y) d y, n \geq 1
$$

where $\varphi$ is a continuous and absolutely integrable function on $\mathbb{R}^{1}$ satisfying $\lim _{|x| \rightarrow \infty} \varphi(x)=0$.

$$
K(n, x)=e^{-\frac{|x|}{\sqrt{D}}} \frac{1}{\sqrt{D}} \sum_{m=0}^{n-1} \frac{n(n+1) \cdots(n+m-1)}{m !(n-1-m) ! 2^{n+m}}\left(\frac{|x|}{\sqrt{D}}\right)^{n-1-m} .
$$

In particular, we use the convention that if $m=0$, then $m !=1$ and $n(n+1) \cdots(n+$ $m-1)=1$. The integral kernel function $K(n, x)$ satisfies the following properties.

\section{Proposition 1 ([1]).}

1. $\int_{-\infty}^{+\infty} K(n, x) d x=1$, for $n>0$;

2. $K(0, x)=\delta(x)$, for $n=0$;

3. For $x \neq 0, n>0, K(n, x)$ is the solution of equation

$$
u(n+1, x)-u(n, x)=D \Delta_{x} u(n+1, x) .
$$

Lemma 2 ([1]). The function $u(n, x)$ is a solution of (15) if, and only if, it is a solution of following non-linear integro-difference equation

$$
u(n+1, x)=\int_{-\infty}^{+\infty} \frac{1}{2 \sqrt{D}} e^{-\frac{|x-y|}{\sqrt{D}}}[u(n, y)+f(u(n, y))] d y, n \geq 0 .
$$

For $\varphi \in C(\mathbf{R}, \mathbf{R})$, define

$$
H(\varphi)(\xi)=(1-d) \varphi(\xi-c)+\eta \int_{-\infty}^{+\infty} b(\varphi(\xi-y-c r)) K_{D_{i}}(r-1, y) d y .
$$

By (H2) and Proposition 1, $H(\varphi)(\xi)$ is well defined. Then, Equation (14) becomes

$$
-D \varphi^{\prime \prime}(\xi)+\varphi(\xi)=H(\varphi)(\xi) .
$$

Let $\Gamma=\left\{\varphi \in C(\mathbb{R}, \mathbb{R}) \mid \varphi\right.$ is non-decerasing in $\xi \in \mathbb{R}, \lim _{\xi \rightarrow-\infty} \varphi(\xi)=0$, $\left.\lim _{\xi \rightarrow+\infty} \varphi(\xi)=w^{*}\right\}$.

For $\varphi, \psi \in \Gamma$, for $\xi \in \mathbb{R}$, we say $\psi \leq \varphi$, if $\psi(\xi) \leq \varphi(\xi)$. Then from the definition of $H$ and Proposition 1, we can easily obtained the following result.

Lemma 3. Assume that (H4) holds. Then, for every $\varphi \in \Gamma, H(\varphi) \in \Gamma$, and $H(\varphi)$ is increasing on $\Gamma$, that is, $H(\psi) \leq H(\varphi)$ for $\varphi, \psi \in \Gamma$ with $\psi \leq \varphi$.

By direct computations, we have 
Lemma 4. For $h \in \Gamma$, the equation

$$
-D \varphi^{\prime \prime}(\xi)+\varphi(\xi)=h(\xi) .
$$

has a unique solution $\varphi \in \Gamma$ satisfying

$$
\varphi(\xi)=\frac{1}{2 \sqrt{D}} \int_{-\infty}^{+\infty} e^{-\frac{|\tilde{s}-\zeta|}{\sqrt{D}}} h(\zeta) d \zeta=\int_{-\infty}^{+\infty} K(1, \xi-\zeta) h(\zeta) d \zeta,
$$

where $K(1, x)$ is defined in (18).

Further define the mapping $\mathbf{F}: \Gamma \rightarrow \Gamma$,

$$
\mathbf{F}(\psi)(\xi)=\int_{-\infty}^{+\infty} K(1, \xi-\zeta) H(\psi)(\zeta) d \zeta, \psi \in \Gamma
$$

The following theorem is a direct consequence of Lemmas 3 and 4 .

Theorem 1. $\varphi \in \Gamma$ is a solution of Equation (14) if, and only if, it satisfies

$$
\varphi(\xi)=\int_{-\infty}^{+\infty} K(1, \xi-\zeta)\left[(1-d) \varphi(\zeta-c)+\eta \int_{-\infty}^{+\infty} b(\varphi(\zeta-y-c r)) K_{D_{i}}(r-1, y) d y\right] d \zeta .
$$

In other words, $\varphi$ is a fixed point of mapping $F$ in $\Gamma$.

Above theorem shows that the existence of traveling wavefronts of Equation (12) is equivalent to the existence of fixed points to mapping $\mathbf{F}$. Therefore, in what follows, we will construct upper and lower solutions of (22) to prove that the mapping $\mathbf{F}$ has a unique fixed point in $\Gamma$, as long as $c$ is greater than a certain constant $c^{*}$.

The linearized equation of (14) at $\varphi=0$ is as below.

$$
\left.-D \varphi^{\prime \prime}(\xi)+\varphi(\xi)=(1-d) \varphi(\xi-c)+\eta \int_{-\infty}^{+\infty} g(0) \varphi(\xi-y-c r)\right) K_{D_{i}}(r-1, y) d y
$$

Then, its characteristic equation is given by

$$
-D \lambda^{2}+1=(1-d) e^{-\lambda c}+g(0) \eta \int_{-\infty}^{+\infty} K_{D_{i}}(r-1, y) e^{-\lambda(y+c r)} d y
$$

Define

$$
\Delta(c, \lambda)=(1-d) e^{-\lambda c}+D \lambda^{2}-1+g(0) \eta \int_{-\infty}^{+\infty} K_{D_{i}}(r-1, y) e^{-\lambda(y+c r)} d y
$$

Note that for $|\lambda|<\frac{1}{\sqrt{D_{i}}}$,

$$
\begin{aligned}
& \int_{-\infty}^{+\infty} K_{D_{i}}(r-1, y) e^{-\lambda y} d y \\
& =\int_{-\infty}^{+\infty} \frac{1}{\sqrt{D_{i}}} e^{-\frac{|y|}{\sqrt{D_{i}}}} \sum_{m=0}^{r-2} \frac{(r-1) \cdot r \cdots(r+m-2)}{m !(r-2-m) ! 2^{r+m-1}}\left(\frac{|y|}{\sqrt{D_{i}}}\right)^{r-2-m} e^{-\lambda y} d y \\
& =\sum_{m=0}^{r-2} \frac{(r-1) \cdot r \cdots(r+m-2)}{m !(r-2-m) ! 2^{r+m-1}} \int_{0}^{+\infty} \frac{1}{\sqrt{\bar{D}_{i}}} e^{-\left(\lambda+\frac{1}{\sqrt{D_{i}}}\right) y}\left(\frac{y}{\sqrt{D_{i}}}\right)^{r-2-m} d y \\
& +\sum_{m=0}^{r-2} \frac{(r-1) \cdot r \cdots(r+m-2)}{m !(r-2-m) ! 2^{r+m-1}} \int_{-\infty}^{0} \frac{1}{\sqrt{D_{i}}} e^{-\left(\lambda-\frac{1}{\sqrt{D_{i}}}\right) y}\left(\frac{-y}{\sqrt{D_{i}}}\right)^{r-2-m} d y \\
& =\sum_{m=0}^{r-2} \frac{(r-1) \cdot r \cdots(r+m-2)}{m !(r-2-m) ! 2^{r+m-1}} \frac{1}{\left(1+\lambda \sqrt{D_{i}}\right)^{r-1-m}} \int_{0}^{+\infty} e^{-z} z^{r-2-m} d z \\
& +\sum_{m=0}^{r-2} \frac{(r-1) \cdot r \cdots(r+m-2)}{m !(r-2-m) ! 2^{r+m-1}} \frac{1}{\left(1-\lambda \sqrt{D_{i}}\right)^{r-1-m}} \int_{0}^{+\infty} e^{-z} z^{r-2-m} d z .
\end{aligned}
$$


Since $\int_{0}^{+\infty} e^{-z} z^{r-2-m} d z=(r-2-m)$ !, we have

$$
\begin{aligned}
& \int_{-\infty}^{+\infty} K_{D_{i}}(r-1, y) e^{-\lambda y} d y \\
= & \sum_{m=0}^{r-2} \frac{(r-1) \cdot r \cdots(r+m-2)}{m ! 2^{r+m-1}}\left[\frac{1}{\left(1+\lambda \sqrt{D_{i}}\right)^{r-1-m}}+\frac{1}{\left(1-\lambda \sqrt{D_{i}}\right)^{r-1-m}}\right] \\
= & \sum_{m=0}^{r-2} \frac{(r-1) \cdot r \cdots(r+m-2)}{m ! 2^{r+m-1}} \cdot \frac{\left(1+\lambda \sqrt{D_{i}}\right)^{r-1-m}+\left(1-\lambda \sqrt{D_{i}}\right)^{r-1-m}}{\left(1-\lambda^{2} D_{i}\right)^{r-1-m}} .
\end{aligned}
$$

By direct computations, we have

$$
\sum_{m=0}^{r-2} \frac{(r-1) \cdot r \cdots(r+m-2)}{m ! 2^{r+m-1}} \cdot \frac{\left(1+\lambda \sqrt{D_{i}}\right)^{r-1-m}+\left(1-\lambda \sqrt{D_{i}}\right)^{r-1-m}}{\left(1-\lambda^{2} D_{i}\right)^{r-1-m}}=\frac{1}{\left(1-\lambda^{2} D_{i}\right)^{r-1}} .
$$

That is,

$$
\int_{-\infty}^{+\infty} K_{D_{i}}(r-1, y) e^{-\lambda y} d y=\frac{1}{\left(1-\lambda^{2} D_{i}\right)^{r-1}} .
$$

In fact, one can give another proof of the equality as follows. By Lemma 1, for $|\lambda|<\frac{1}{\sqrt{D_{i}}}$, the initial value problem

$$
\left\{\begin{array}{l}
u(n+1, x)-u(n, x)=D_{i} \Delta_{x} u(n+1, x), x \in \mathbb{R}, n \geq 0 \\
u(0, x)=e^{-\lambda x}
\end{array}\right.
$$

has a unique solution $u(n, x)$ and

$$
u(n, x)=\int_{-\infty}^{\infty} K_{D_{i}}(n, x-y) e^{-\lambda y} d y, n \geq 1
$$

On the other hand, one can easily verified that

$$
u(n, x)=\frac{e^{-\lambda x}}{\left(1-\lambda^{2} D_{i}\right)^{n}}
$$

is a solution to initial value problem (26). Therefore,

$$
\int_{-\infty}^{\infty} K_{D_{i}}(n, x-y) e^{-\lambda y} d y=\frac{e^{-\lambda x}}{\left(1-\lambda^{2} D_{i}\right)^{n}}
$$

which leads to the equality (25). Then

$$
\Delta(c, \lambda)=(1-d) e^{-\lambda c}+D \lambda^{2}-1+\frac{g(0) \eta e^{-\lambda c r}}{\left(1-\lambda^{2} D_{i}\right)^{r-1}}
$$

and

$$
\begin{aligned}
& \Delta_{\lambda}^{\prime}(c, \lambda)=-c(1-d) e^{-\lambda c}+2 D \lambda+g(0) \eta \frac{e^{-\lambda c r}\left[2 \lambda(r-1) D_{i}-c r\left(1-\lambda^{2} D_{i}\right)\right]}{\left(1-\lambda^{2} D_{i}\right)^{r}}, \\
& \Delta_{\lambda}^{\prime \prime}(c, \lambda)=c^{2}(1-d) e^{-\lambda c}+2 D+g(0) \eta \frac{e^{-\lambda c r}}{\left(1-\lambda^{2} D_{i}\right)^{r+1}}\left\{\left[2 \lambda(r-1) D_{i}-c r\left(1-\lambda^{2} D_{i}\right)\right]^{2}\right. \\
& \left.+2(r-1) D_{i}\left(1-\lambda^{2} D_{i}\right)+4 \lambda^{2} D_{i}^{2}(r-1)\right\} .
\end{aligned}
$$

It is easy to see that for any given $c \geq 0$ and $|\lambda|<\frac{1}{\sqrt{D_{i}}}, \Delta_{\lambda}^{\prime \prime}(c, \lambda)>0$. Then, for any given $c \geq 0, \Delta_{\lambda}^{\prime}(c, \lambda)$ strictly increasing in $\lambda \in\left(0, \frac{1}{\sqrt{D_{i}}}\right)$. Additionally, from 


$$
\Delta_{\lambda}^{\prime}(c, 0)=-c(1-d)-g(0) \eta c r<0, \forall c>0
$$

and $\lim _{\lambda \rightarrow \frac{1}{\sqrt{D_{i}}}-0} \Delta_{\lambda}^{\prime}(c, \lambda)=+\infty$, for any $c>0$, there exists unique $\lambda=\lambda(c), 0<\lambda(c)<\frac{1}{\sqrt{D_{i}}}$, such that $\Delta_{\lambda}^{\prime}(c, \lambda(c))=0$ and

$$
\Delta_{\text {min }}=\Delta(c, \lambda(c))=(1-d) e^{-\lambda(c) c}+D \lambda^{2}(c)-1+\frac{g(0) \eta e^{-\lambda(c) c r}}{\left(1-\lambda^{2}(c) D_{i}\right)^{r-1}} .
$$

If $\Delta(c, \lambda(c))>0$, the characteristic Equation (24) has no positive real roots; If $\Delta(c, \lambda(c))=0$, the characteristic Equation (24) has only one positive real root; If $\Delta(c, \lambda(c))<0$, the characteristic Equation (24) has exactly two positive real roots.

Lemma 5. There exist a positive constant $c^{*}>0$, such that

(i) If $c>c^{*}$, then the characteristic Equation (24) has two positive real roots, $0<\lambda_{1}(c)<\lambda_{2}(c)$;

(ii) If $c<c^{*}$, then the characteristic Equation (24) has no positive real roots;

(iii) If $c=c^{*}$, the characteristic Equation (24) has only one positive real roots.

Proof. Consider the function $\Delta(c, \lambda)=(1-d) e^{-\lambda c}+D \lambda^{2}-1+g(0) \eta \frac{e^{-\lambda c r}}{\left(1-\lambda^{2} D_{i}\right)^{r-1}}$. When $c=0$ and $0 \leq \lambda<\frac{1}{\sqrt{D_{i}}}$, due to $g(0) \eta>d$, we have

$$
\Delta(0, \lambda)=(1-d)+D \lambda^{2}-1+\frac{g(0) \eta}{\left(1-\lambda^{2} D_{i}\right)^{r-1}}>D \lambda^{2}-d+g(0) \eta>g(0) \eta-d>0
$$

which implies that (24) will has no positive real roots.

By the continuity of $\Delta(c, \lambda)$ in $c$, for sufficiently small $c>0,(24)$ will also have no positive real roots. Otherwise, there will be sequences $\left\{c_{n}\right\}$ and $\lambda_{n}$ with $c_{n}>0, \lim _{n \rightarrow \infty} c_{n}=0$ and $0<\lambda_{n}<\frac{1}{\sqrt{D_{i}}}$, such that $\Delta\left(c_{n}, \lambda_{n}\right)=0$, i.e.,

$$
(1-d) e^{-\lambda_{n} c_{n}}+D \lambda_{n}^{2}-1+\frac{g(0) \eta e^{-\lambda_{n} c_{n} r}}{\left(1-\lambda_{n}^{2} D_{i}\right)^{r-1}}=0
$$

Clearly, there exists a convergent subsequence $\left\{\lambda_{n_{k}}\right\}$, and let $\lim _{k \rightarrow \infty} \lambda_{n_{k}}=\lambda^{*}$. If $\lambda^{*} \in\left[0, \frac{1}{\sqrt{D_{i}}}\right)$, then,

$$
0=(1-d)+D \lambda^{* 2}-1+\frac{g(0) \eta}{\left(1-\lambda^{* 2} D_{i}\right)^{r-1}}>D \lambda^{* 2}+g(0) \eta-d>0
$$

Contradiction. If $\lambda^{*}=\frac{1}{\sqrt{D_{i}}}$, then

$$
0=(1-d)+\frac{D}{D_{i}}-1+\lim _{k \rightarrow \infty} \frac{g(0) \eta}{\left(1-\lambda_{n_{k}}^{2} D_{i}\right)^{r-1}}=\infty
$$

It is also a contradiction.

Next, we consider $\Delta_{c}^{\prime}(c, \lambda)$ for any given $\lambda \in\left(0, \min \left\{\frac{1}{\sqrt{D}}, \frac{1}{\sqrt{D_{i}}}\right\}\right)$,

$$
\Delta_{c}^{\prime}(c, \lambda)=-(1-d) \lambda e^{-\lambda c}-\lambda r g(0) \eta \frac{e^{-\lambda c r}}{\left(1-\lambda^{2} D_{i}\right)^{r-1}}<0 .
$$

Since $\Delta(0, \lambda)>0, \lim _{c \rightarrow+\infty} \Delta(c, \lambda)=D \lambda^{2}-1<0$, then there exists unique $c=c(\lambda)$, such that $\Delta(c(\lambda), \lambda)=0$. Denote $c^{*}=\min \left\{c(\lambda) \mid \lambda \in\left(0, \min \left\{\frac{1}{\sqrt{D}}, \frac{1}{\sqrt{D_{i}}}\right\}\right)\right\}$. Obviously $c^{*}>0$. Moreover, $\Delta\left(c^{*}, \lambda\right)=0$ has exactly one positive real root and $\Delta\left(c^{*}, \lambda\right)$ attains its minimum at the point $\lambda=\lambda\left(c^{*}\right)$. This completes the proof. 
In what follows, we will consider the case where $c>c^{*}$. In this case, the characteristic Equation (24) has two positive real roots $\lambda_{1}(c)$ and $\lambda_{2}(c)$ satisfying $0<\lambda_{1}(c)<\lambda_{2}(c)<$ $\min \left\{\frac{1}{\sqrt{D}}, \frac{1}{\sqrt{D_{i}}}\right\}$ and $\Delta(c, \lambda)<0$ for $\lambda \in\left(\lambda_{1}(c), \lambda_{2}(c)\right)$.

Definition 1. A continuous bounded function $\phi: \mathbb{R} \rightarrow\left[0, w^{*}\right]$ is called an upper solution of (22) if it satisfies

$$
\phi(\xi) \geq \int_{-\infty}^{+\infty} K(1, \xi-\zeta)\left[(1-d) \phi(\zeta-c)+\eta \int_{-\infty}^{+\infty} b(\phi(\zeta-y-c r)) K_{D_{i}}(r-1, y) d y\right] d \zeta,
$$

A lower solution of (22) is defined in a similar way by reversing the inequality in (29).

Now fixed $c>c^{*}$ and let $0<\lambda_{1}(c)<\lambda_{2}(c)<\min \left\{\frac{1}{\sqrt{D}}, \frac{1}{\sqrt{D_{i}}}\right\}$. Choose sufficiently large constant $q>1$ to be determined later, define the functions $\bar{\phi}$ and $\phi$ by

$$
\bar{\phi}(\xi)=\min \left\{w^{*}, w^{*} e^{\lambda_{1}(c) \xi}\right\}, \underline{\phi}(\xi)=\max \left\{0, w^{*}\left[e^{\lambda_{1}(c) \xi}-q e^{p \lambda_{1}(c) \xi}\right]\right\},
$$

where $p \in\left(1, \min \left\{2, \frac{\lambda_{2}(c)}{\lambda_{1}(c)}\right\}\right), w^{*}$ satisfies $\eta g\left(w^{*}\right)=d$.

Proposition 2. $\bar{\phi}(\xi)$ is an upper solution of (22).

Proof. By the definition of $\bar{\phi}(\xi)$, for any $\xi \in \mathbb{R}, \bar{\phi}(\xi) \leq w^{*}$. Thus, by (H4) and Proposition 1 , we achieve

$$
\begin{aligned}
& \int_{-\infty}^{+\infty} K(1, \xi-\zeta)\left[(1-d) \bar{\phi}(\zeta-c)+\eta \int_{-\infty}^{+\infty} b(\bar{\phi}(\zeta-y-c r)) K_{D_{i}}(r-1, y) d y\right] d \zeta \\
\leq & \int_{-\infty}^{+\infty} K(1, \xi-\zeta)\left[(1-d) w^{*}+\eta b\left(w^{*}\right)\right] d \zeta \\
\leq & w^{*} \int_{-\infty}^{+\infty} K(1, \xi-\zeta) d \zeta=w^{*} .
\end{aligned}
$$

For $\xi \in \mathbb{R}$, if $\bar{\phi}(\xi)=w^{*}$, clearly, we have,

$$
\int_{-\infty}^{+\infty} K(1, \xi-\zeta)\left[(1-d) \bar{\phi}(\zeta-c)+\eta \int_{-\infty}^{+\infty} b(\bar{\phi}(\zeta-y-c r)) K_{D_{i}}(r-1, y) d y\right] d \zeta \leq \bar{\phi}(\xi) .
$$

If $\bar{\phi}(\xi)=w^{*} e^{\lambda_{1}(c) \xi}$, that is, $\xi \leq 0$, by $(\mathrm{H} 1)$ and the definition of $\bar{\phi}(\xi)$,

$$
\begin{aligned}
& \int_{-\infty}^{+\infty} K(1, \xi-\zeta)\left[(1-d) \bar{\phi}(\zeta-c)+\eta \int_{-\infty}^{+\infty} b(\bar{\phi}(\zeta-y-c r)) K_{D_{i}}(r-1, y) d y\right] d \zeta \\
& \leq \int_{-\infty}^{+\infty} K(1, \xi-\zeta)\left[(1-d) \bar{\phi}(\zeta-c)+\eta g(0) \int_{-\infty}^{+\infty} \bar{\phi}(\zeta-y-c r) K_{D_{i}}(r-1, y) d y\right] d \zeta \\
& \leq \int_{-\infty}^{+\infty} K(1, \xi-\zeta)\left[(1-d) w^{*} e^{\lambda_{1}(c)(\zeta-c)}+\eta g(0) \int_{-\infty}^{+\infty} w^{*} e^{\lambda_{1}(c)(\zeta-y-c r)} K_{D_{i}}(r-1, y) d y\right] d \zeta \\
& =w^{*} \int_{-\infty}^{+\infty} K(1, \xi-\zeta) e^{\lambda_{1}(c) \zeta}\left[(1-d) e^{-\lambda_{1}(c) c}+\eta g(0) \int_{-\infty}^{+\infty} e^{-\lambda_{1}(c)(y+c r)} K_{D_{i}}(r-1, y) d y\right] d \zeta \\
& =w^{*}\left[1-D \lambda_{1}^{2}(c)\right] \int_{-\infty}^{+\infty} K(1, \xi-\zeta) e^{\lambda_{1}(c) \zeta} d \zeta .
\end{aligned}
$$

The last equality follows from the fact that $\lambda_{1}(c)$ is a root of $\Delta(c, \lambda)$. Calculate above integral directly, 


$$
\begin{aligned}
& \int_{-\infty}^{+\infty} K(1, \xi-\zeta) e^{\lambda_{1}(c) \zeta} d \zeta \\
= & \frac{1}{2 \sqrt{D}} \int_{-\infty}^{+\infty} e^{-\frac{|\tilde{\xi}-\zeta|}{\sqrt{D}}} e^{\lambda_{1}(c) \zeta} d \zeta \\
= & \frac{1}{2 \sqrt{D}} \int_{\xi}^{+\infty} e^{-\frac{\zeta-\xi}{\sqrt{D}}} e^{\lambda_{1}(c) \zeta} d \zeta+\frac{1}{2 \sqrt{D}} \int_{-\infty}^{\xi} e^{-\frac{\xi-\zeta}{\sqrt{D}}} e^{\lambda_{1}(c) \zeta} d \zeta \\
= & -\frac{e^{\frac{\xi}{\sqrt{D}}}}{2 \sqrt{D}\left[\lambda_{1}(c)-\frac{1}{\sqrt{D}}\right]} e^{\left[\lambda_{1}(c)-\frac{1}{\sqrt{D}}\right] \xi}+\frac{e^{-\frac{\xi}{\sqrt{D}}}}{2 \sqrt{D}\left[\lambda_{1}(c)+\frac{1}{\sqrt{D}}\right]} e^{\left[\lambda_{1}(c)+\frac{1}{\sqrt{D}}\right] \xi} \\
= & \frac{1}{1-D \lambda_{1}^{2}(c)} e^{\lambda_{1}(c) \xi} .
\end{aligned}
$$

It follows that,

$$
\int_{-\infty}^{+\infty} K(1, \xi-\zeta)\left[(1-d) \bar{\phi}(\zeta-c)+\eta \int_{-\infty}^{+\infty} b(\bar{\phi}(\zeta-y-c r)) K_{D_{i}}(r-1, y) d y\right] d \zeta \leq \bar{\phi}(\xi) .
$$

This proves that $\bar{\phi}(\xi)$ is an upper solution of (22).

Proposition 3. For any given $p \in\left(1, \min \left\{2, \frac{\lambda_{2}(c)}{\lambda_{1}(c)}\right\}\right)$, there exist a sufficiently large positive constant $q>1$, such that $\underline{\phi}(\xi)$ is a lower solution of (22).

Proof. Let

$$
\kappa(\xi)=w^{*}\left[e^{\lambda_{1}(c) \xi}-q e^{p \lambda_{1}(c) \xi}\right]
$$

Then

$$
\mathcal{K}^{\prime}(\xi)=w^{*} \lambda_{1}(c)\left[e^{\lambda_{1}(c) \xi}-q p e^{p \lambda_{1}(c) \xi}\right] .
$$

There must exist $\xi_{0}=\frac{\ln q}{\lambda_{1}(c)(1-p)}<0$, such that $\kappa\left(\xi_{0}\right)=0$ and $\xi_{1}=\frac{\ln p q}{\lambda_{1}(c)(1-p)}<\xi_{0}$, such that $\mathcal{K}^{\prime}\left(\xi_{1}\right)=0$. We know that $\kappa$ is increasing on $\left(-\infty, \xi_{1}\right)$ and decreasing on $\left(\xi_{1},+\infty\right)$, and

$$
\kappa\left(\xi_{1}\right)=w^{*} \frac{p-1}{p} \frac{1}{(p q)^{1 /(p-1)}} \in\left(0, w^{*}\right) .
$$

Consequently, $0 \leq \underline{\phi}(\xi)<w^{*}$, for $\xi \in \mathbb{R}$.

If $\underline{\phi}(\xi)=0$,

$$
\begin{aligned}
& \int_{-\infty}^{+\infty} K(1, \xi-\zeta)\left[(1-d) \underline{\phi}(\zeta-c)+\eta \int_{-\infty}^{+\infty} b(\underline{\phi}(\zeta-y-c r)) K_{D_{i}}(r-1, y) d y\right] d \zeta \geq 0=\underline{\phi}(\xi) . \\
& \text { If } \underline{\phi}(\xi)=w^{*}\left[e^{\lambda_{1}(c) \xi}-q e^{\left.p \lambda_{1}(c) \xi\right]},\right. \\
& \quad \int_{-\infty}^{+\infty} K(1, \xi-\zeta)\left[(1-d) \underline{\phi}(\zeta-c)+\eta \int_{-\infty}^{+\infty} b(\underline{\phi}(\zeta-y-c r)) K_{D_{i}}(r-1, y) d y\right] d \zeta \\
& \quad=\int_{-\infty}^{+\infty} K(1, \xi-\zeta)\left[(1-d) \underline{\phi}(\zeta-c)+\eta \int_{-\infty}^{+\infty} \underline{\phi}(\zeta-y-c r) g(\underline{\phi}(\zeta-y-c r)) K_{D_{i}}(r-1, y) d y\right] d \zeta \\
& \quad=\int_{-\infty}^{+\infty} K(1, \xi-\zeta)\left[(1-d) \underline{\phi}(\zeta-c)+\eta \int_{-\infty}^{+\infty} \underline{\phi}(\zeta-y-c r) g(0) K_{D_{i}}(r-1, y) d y\right] d \zeta \\
& \quad+\eta \int_{-\infty}^{+\infty} K(1, \xi-\zeta) \int_{-\infty}^{+\infty} \underline{\phi}(\zeta-y-c r)[g(\underline{\phi}(\zeta-y-c r))-g(0)] K_{D_{i}}(r-1, y) d y d \zeta:=I_{1}+I_{2}
\end{aligned}
$$

where

$$
I_{1}=\int_{-\infty}^{+\infty} K(1, \xi-\zeta)\left[(1-d) \underline{\phi}(\zeta-c)+\eta \int_{-\infty}^{+\infty} \underline{\phi}(\zeta-y-c r) g(0) K_{D_{i}}(r-1, y) d y\right] d \zeta
$$




$$
I_{2}=\eta \int_{-\infty}^{+\infty} K(1, \xi-\zeta) \int_{-\infty}^{+\infty} \underline{\phi}(\zeta-y-c r)[g(\underline{\phi}(\zeta-y-c r))-g(0)] K_{D_{i}}(r-1, y) d y d \zeta
$$

For $I_{1}$, we have

$$
\begin{aligned}
I_{1} & \geq \int_{-\infty}^{+\infty} K(1, \xi-\zeta)\left\{(1-d) w^{*}\left[e^{\lambda_{1}(c)(\zeta-c)}-q e^{p \lambda_{1}(c)(\zeta-c)}\right]\right. \\
& \left.+\eta g(0) \int_{-\infty}^{+\infty} w^{*}\left[e^{\lambda_{1}(c)(\zeta-y-c r)}-q e^{p \lambda_{1}(c)(\zeta-y-c r)}\right] K_{D_{i}}(r-1, y) d y\right\} d \zeta \\
& =w^{*} \int_{-\infty}^{+\infty} K(1, \xi-\zeta)\left[(1-d) e^{\lambda_{1}(c)(\zeta-c)}+g(0) \eta \int_{-\infty}^{+\infty} e^{\lambda_{1}(c)(\zeta-y-c r)} K_{D_{i}}(r-1, y) d y\right] d \zeta \\
& -w^{*} q \int_{-\infty}^{+\infty} K(1, \xi-\zeta)\left[(1-d) e^{p \lambda_{1}(c)(\zeta-c)}+g(0) \eta \int_{-\infty}^{+\infty} e^{p \lambda_{1}(c)(\zeta-y-c r)} K_{D_{i}}(r-1, y) d y\right] d \zeta \\
& =w^{*}\left[(1-d) e^{-\lambda_{1}(c) c}+g(0) \eta \int_{-\infty}^{+\infty} e^{-\lambda_{1}(c)(y+c r)} K_{D_{i}}(r-1, y) d y\right] \int_{-\infty}^{+\infty} K(1, \xi-\zeta) e^{\lambda_{1}(c) \zeta} d \zeta \\
& -w^{*} q\left[(1-d) e^{-p \lambda_{1}(c) c}+g(0) \eta \int_{-\infty}^{+\infty} e^{-p \lambda_{1}(c)(y+c r)} K_{D_{i}}(r-1, y) d y\right] \int_{-\infty}^{+\infty} K(1, \xi-\zeta) e^{p \lambda_{1}(c) \zeta} d \zeta .
\end{aligned}
$$

Note that,

$$
\begin{gathered}
\Delta\left(c, \lambda_{1}(c)\right)=(1-d) e^{-\lambda_{1}(c) c}+g(0) \eta \int_{-\infty}^{+\infty} e^{-\lambda_{1}(c)(y+c r)} K_{D_{i}}(r-1, y) d y+D \lambda_{1}^{2}(c)-1=0, \\
\Delta\left(c, p \lambda_{1}(c)\right)=(1-d) e^{-p \lambda_{1}(c) c}+g(0) \eta \int_{-\infty}^{+\infty} e^{-p \lambda_{1}(c)(y+c r)} K_{D_{i}}(r-1, y) d y+D p^{2} \lambda_{1}^{2}(c)-1 .
\end{gathered}
$$

Therefore,

$$
\begin{aligned}
I_{1} & \geq w^{*}\left[1-D \lambda_{1}^{2}(c)\right] \int_{-\infty}^{+\infty} K(1, \xi-\zeta) e^{\lambda_{1}(c) \zeta} d \zeta \\
& \quad-w^{*} q\left[\Delta\left(c, p \lambda_{1}(c)\right)+1-D p^{2} \lambda_{1}^{2}(c)\right] \int_{-\infty}^{+\infty} K(1, \xi-\zeta) e^{p \lambda_{1}(c) \zeta} d \zeta
\end{aligned}
$$

From the proof of Proposition 2, we know

$$
\int_{-\infty}^{+\infty} K(1, \xi-\zeta) e^{\lambda_{1}(c) \zeta} d \zeta=\frac{1}{1-D \lambda_{1}^{2}(c)} e^{\lambda_{1}(c) \xi}
$$

and similar arguments have

$$
\int_{-\infty}^{+\infty} K(1, \xi-\zeta) e^{p \lambda_{1}(c) \zeta} d \zeta=\frac{1}{1-D p^{2} \lambda_{1}^{2}(c)} e^{p \lambda_{1}(c) \xi} .
$$

Therefore,

$$
\begin{aligned}
I_{1} & \geq w^{*}\left[e^{\lambda_{1}(c) \xi}-q e^{p \lambda_{1}(c) \xi}\right]-w^{*} q \Delta\left(c, p \lambda_{1}(c)\right) \frac{1}{1-D p^{2} \lambda_{1}^{2}(c)} e^{p \lambda_{1}(c) \xi} \\
& \geq \underline{\phi}(\xi)-\frac{w^{*} q \Delta\left(c, p \lambda_{1}(c)\right)}{1-D p^{2} \lambda_{1}^{2}(c)} e^{p \lambda_{1}(c) \xi} .
\end{aligned}
$$

Since $\lambda_{1}(c)<p \lambda_{1}(c)<\lambda_{2}(c)<\frac{1}{\sqrt{D}}, \Delta\left(c, p \lambda_{1}(c)\right)<0$, we have

$$
-\frac{w^{*} q \Delta\left(c, p \lambda_{1}(c)\right)}{1-D p^{2} \lambda_{1}^{2}(c)}>0 .
$$

For $I_{2}$, by $(\mathrm{H} 1)$, we know $g^{\prime}(w) \leq 0$. Let $-L \leq g^{\prime}(w) \leq 0, \forall w \in\left[0, w^{*}\right]$. Then 


$$
\begin{aligned}
I_{2} & =\eta \int_{-\infty}^{+\infty} K(1, \xi-\zeta) \int_{-\infty}^{+\infty} \underline{\phi}(\zeta-y-c r)[g(\underline{\phi}(\zeta-y-c r))-g(0)] K_{D_{i}}(r-1, y) d y d \zeta \\
& =\eta \int_{-\infty}^{+\infty} K(1, \xi-\zeta) \int_{-\infty}^{+\infty} \underline{\phi}^{2}(\zeta-y-c r) g^{\prime}(\theta \underline{\phi}(\zeta-y-c r)) K_{D_{i}}(r-1, y) d y d \zeta \quad(\theta \in(0,1)) \\
& \geq-L \eta \int_{-\infty}^{+\infty} K(1, \xi-\zeta) \int_{-\infty}^{+\infty} \underline{\phi^{2}}(\zeta-y-c r) K_{D_{i}}(r-1, y) d y d \zeta .
\end{aligned}
$$

According to the definition of $\xi_{0}$,

$$
\begin{aligned}
& \int_{-\infty}^{+\infty} K(1, \xi-\zeta) \int_{-\infty}^{+\infty} \underline{\phi}^{2}(\zeta-y-c r) K_{D_{i}}(r-1, y) d y d \zeta \\
= & \left(w^{*}\right)^{2} \int_{-\infty}^{+\infty} K(1, \xi-\zeta) \int_{\zeta-c r-\xi_{0}}^{+\infty}\left[e^{\lambda_{1}(c)(\zeta-y-c r)}-q e^{p \lambda_{1}(c)(\zeta-y-c r)}\right]^{2} K_{D_{i}}(r-1, y) d y d \zeta \\
\leq & \left(w^{*}\right)^{2} \int_{-\infty}^{+\infty} K(1, \xi-\zeta) \int_{\zeta-c r-\xi_{0}}^{+\infty} e^{2 \lambda_{1}(c)(\zeta-y-c r)} K_{D_{i}}(r-1, y) d y d \zeta \\
\leq & \left(w^{*}\right)^{2} \int_{-\infty}^{+\infty} K(1, \xi-\zeta) \int_{\zeta-c r-\xi_{0}}^{+\infty} e^{p \lambda_{1}(c)(\zeta-y-c r)} K_{D_{i}}(r-1, y) d y d \zeta \\
= & \left(w^{*}\right)^{2} \int_{-\infty}^{+\infty} K(1, \xi-\zeta) e^{p \lambda_{1}(c)(\zeta-c r)} \int_{\zeta-c r-\xi_{0}}^{+\infty} e^{-p \lambda_{1}(c) y} K_{D_{i}}(r-1, y) d y d \zeta \\
\leq & \left(w^{*}\right)^{2} \int_{-\infty}^{+\infty} K(1, \xi-\zeta) e^{p \lambda_{1}(c)(\zeta-c r)} \int_{-\infty}^{+\infty} e^{-p \lambda_{1}(c) y} K_{D_{i}}(r-1, y) d y d \zeta .
\end{aligned}
$$

Due to (25),

$$
\int_{-\infty}^{+\infty} e^{-p \lambda_{1}(c) y} K_{D_{i}}(r-1, y) d y=\frac{1}{\left(1-p^{2} \lambda_{1}^{2}(c) D_{i}\right)^{r-1}} .
$$

Then

$$
\begin{aligned}
& \int_{-\infty}^{+\infty} K(1, \xi-\zeta) \int_{-\infty}^{+\infty} \underline{\phi}^{2}(\zeta-y-c r) K_{D_{i}}(r-1, y) d y d \zeta \\
\leq & \frac{\left(w^{*}\right)^{2}}{\left(1-p^{2} \lambda_{1}^{2}(c) D_{i}\right)^{r-1}} \int_{-\infty}^{+\infty} K(1, \xi-\zeta) e^{p \lambda_{1}(c)(\zeta-c r)} d \zeta \\
\leq & \frac{\left(w^{*}\right)^{2}}{\left(1-p^{2} \lambda_{1}^{2}(c) D_{i}\right)^{r-1}} e^{-p \lambda_{1}(c) c r} \int_{-\infty}^{+\infty} K(1, \xi-\zeta) e^{p \lambda_{1}(c) \zeta} d \zeta \\
= & \frac{\left(w^{*}\right)^{2}}{\left(1-p^{2} \lambda_{1}^{2}(c) D_{i}\right)^{r-1}} e^{-p \lambda_{1}(c) c r} \frac{1}{1-D p^{2} \lambda_{1}^{2}(c)} e^{p \lambda_{1}(c) \xi} .
\end{aligned}
$$

Thus,

$$
I_{2} \geq-L \eta \frac{\left(w^{*}\right)^{2} e^{p \lambda_{1}(c)(\xi-c r)}}{\left[1-p^{2} \lambda_{1}^{2}(c) D_{i}\right]^{r-1}\left[1-D p^{2} \lambda_{1}^{2}(c)\right]} .
$$

Based on the above results,

$$
\begin{aligned}
I_{1}+I_{2} & \geq \underline{\phi}(\xi)-\frac{w^{*} q \Delta\left(c, p \lambda_{1}(c)\right)}{1-D p^{2} \lambda_{1}^{2}(c)} e^{p \lambda_{1}(c) \xi}-L \eta \frac{\left(w^{*}\right)^{2} e^{p \lambda_{1}(c)(\xi-c r)}}{\left[1-p^{2} \lambda_{1}^{2}(c) D_{i}\right]^{r-1}\left[1-D p^{2} \lambda_{1}^{2}(c)\right]} \\
& =\underline{\phi}(\xi)+\frac{w^{*} e^{p \lambda_{1}(c) \xi}}{1-D p^{2} \lambda_{1}^{2}(c)}\left[-q \Delta\left(c, p \lambda_{1}(c)\right)-\frac{L \eta w^{*} e^{-p \lambda_{1}(c) c r}}{\left[1-p^{2} \lambda_{1}^{2}(c) D_{i}\right]^{r-1}}\right] .
\end{aligned}
$$

Since $\Delta\left(c, p \lambda_{1}(c)\right)<0$, when $q>1$ sufficiently large, 


$$
-q \Delta\left(c, p \lambda_{1}(c)\right)-\frac{L \eta w^{*} e^{-p \lambda_{1}(c) c r}}{\left[1-p^{2} \lambda_{1}^{2}(c) D_{i}\right]^{r-1}}>0 .
$$

Hence, for such $q$,

$$
I_{1}+I_{2} \geq \underline{\phi}(\xi)
$$

That is, $\underline{\phi}(\xi)$ is a lower solution of (22). This completes the proof.

We have already obtained an upper and a lower solution of (22). Using the classical upper and lower solution method together with iteration techniques, we find the following existence result.

Theorem 2. Assume that (H1)-(H4) hold. Then for any $c>c^{*},(12)$ admits a traveling wavefront solution $w(n, x)=\varphi(x+c n)$ connecting the equilibrium 0 and $w^{*}$.

\section{Traveling Wave Solution for the Non-Monotone Case}

This section is devoted to the existence of traveling wave solutions to (1) when the birth function $b(w)$ is not increasing in $\left[0, w^{*}\right]$. Our approach is to construct two auxiliary temporally discrete diffusion equations with birth functions satisfying monotonic conditions. Then, by using a similar method developed in [23] and applying the results obtained in the Section 1 to these auxiliary equations, we can prove that (1) possesses a traveling wave solution connecting equilibrium 0 and $w^{*}$.

Throughout of this section, we suppose that $b(w)$ satisfies assumptions (H1)-(H3).

By (H3) and the continuity of $g(w)$ at zero, there exists a small constant $\delta>0$, such that for any $w \in(0, \delta), g(w)>\frac{d}{\eta}$. Then, for any $w \in(0, \delta), b(w)-\frac{d}{\eta} w=w\left(g(w)-\frac{d}{\eta}\right)>0$. For such $w, \max \{b(z) \mid 0 \leq z \leq w\}>\frac{d}{\eta} w$. Then

On the other hand, when $w>w^{*}, g(w)<\frac{d}{\eta}$. This means that for $w>w^{*}, b(w)-\frac{d}{\eta} w<0$.

$$
\max \left\{b(z) \mid w^{*}<z \leq w\right\}<\frac{d}{\eta} w .
$$

Let $W^{*}=\frac{\eta}{d} \max \left\{b(w) \mid 0 \leq w \leq w^{*}\right\}$. Then $W^{*} \geq w^{*}$. For any $w \in\left(w^{*}, W^{*}\right]$, $b(w) \leq \frac{d}{\eta} w \leq \frac{d}{\eta} W^{*}$. Consequently,

$$
\frac{d W^{*}}{\eta} \geq \max \left\{b(w) \mid 0 \leq w \leq W^{*}\right\}
$$

Let $W_{*}=\frac{\eta}{d} \min \left\{b(w) \mid w^{*} \leq w \leq W^{*}\right\}$. Then $W_{*}>0$. Clearly, $0<W_{*} \leq w^{*}$ and $b(w)>\frac{d w}{\eta}$ for all $w \in\left(0, W_{*}\right)$.

We note that, if $b\left(w^{*}\right)=\max \left\{b(w) \mid 0 \leq w \leq w^{*}\right\}$, then $W^{*}=W_{*}=w^{*}$. For sufficiently small $\varepsilon<W_{*}$, we define two auxiliary functions $b^{*}(w)$ and $b_{\epsilon}(w)$ as follows.

$$
b^{*}(w)= \begin{cases}b^{\prime}(0) w, & \text { for } w \in\left[0, \frac{d}{b^{\prime}(0) \eta} W^{*}\right], \\ \frac{d W^{*}}{\eta}, & \text { for } w \in\left(\frac{d}{b^{\prime}(0) \eta} W^{*}, W^{*}\right] \\ \max \left\{\frac{d W^{*}}{\eta}, b(w)\right\}, & \text { for } w>W^{*}\end{cases}
$$

and

$$
b_{\varepsilon}(w)= \begin{cases}\inf _{s \in\left[w, W^{*}\right]}\left\{b(s), \frac{d\left(W_{*}-\varepsilon\right)}{\eta}\right\}, & \text { for } w \in\left[0, W^{*}\right], \\ \min \left\{b(w), \frac{d\left(W_{*}-\varepsilon\right)}{\eta}\right\}, & \text { for } w>W^{*}\end{cases}
$$

Lemma 6. The following statements hold true:

(i) $b^{*}$ and $b_{\varepsilon}$ are continuous on $[0,+\infty)$ and non-decreasing on $\left[0, W^{*}\right]$;

(ii) $b^{\prime}(0) w \geq b^{*}(w) \geq b(w) \geq b_{\varepsilon}(w)>0$ for all $w>0$; 
(iii) $b^{*}(0)=0, b^{*}\left(W^{*}\right)=\frac{d}{\eta} W^{*}$ and $b^{*}(w)>\frac{d}{\eta} w$ for all $w \in\left(0, W^{*}\right)$;

(iv) $b_{\varepsilon}(0)=0, b_{\varepsilon}\left(W_{*}-\varepsilon\right)=\frac{d}{\eta}\left(W_{*}-\varepsilon\right)$ and $b_{\varepsilon}(w)>\frac{d}{\eta} w$ for all $w \in\left(0, W_{*}-\varepsilon\right)$;

(v) $\left(b^{*}\right)^{\prime}(0)=b_{\varepsilon}^{\prime}(0)=b^{\prime}(0)$.

The proof of Lemma is a direct verification, we omit it.

Now we consider the following two auxiliary temporally discrete diffusion equations

$$
\begin{aligned}
& w(n+1, x)=D \Delta w(n+1, x)+(1-d) w(n, x)+\eta \int_{-\infty}^{+\infty} K_{D_{i}}(r-1, x-y) b^{*}(w(n+1-r, y)) d y \\
& \quad \text { and } \\
& w(n+1, x)=D \Delta w(n+1, x)+(1-d) w(n, x)+\eta \int_{-\infty}^{+\infty} K_{D_{i}}(r-1, x-y) b_{\varepsilon}(w(n+1-r, y)) d y .
\end{aligned}
$$

Then, the corresponding wave equations of (30) and (31) read as

$$
-D \varphi^{\prime \prime}(\xi)+\varphi(\xi)=(1-d) \varphi(\xi-c)+\eta \int_{-\infty}^{+\infty} K_{D_{i}}(r-1, y) b^{*}(\varphi(\xi-y-c r)) d y
$$

and

$$
-D \varphi^{\prime \prime}(\xi)+\varphi(\xi)=(1-d) \varphi(\xi-c)+\eta \int_{-\infty}^{+\infty} K_{D_{i}}(r-1, y) b_{\varepsilon}(\varphi(\xi-y-c r)) d y,
$$

respectively. They are equivalent to the following two integral equations:

$$
\begin{aligned}
& \varphi(\xi)=\int_{-\infty}^{+\infty} K(1, \xi-\zeta)\left[(1-d) \varphi(\zeta-c)+\eta \int_{-\infty}^{+\infty} b^{*}(\varphi(\zeta-y-c r)) K_{D_{i}}(r-1, y) d y\right] d \zeta, \\
& \varphi(\xi)=\int_{-\infty}^{+\infty} K(1, \xi-\zeta)\left[(1-d) \varphi(\zeta-c)+\eta \int_{-\infty}^{+\infty} b_{\varepsilon}(\varphi(\zeta-y-c r)) K_{D_{i}}(r-1, y) d y\right] d \zeta .
\end{aligned}
$$

Moreover, the traveling wavefronts of (30) and (31) are fixed points of operators $F^{*}$ and $F_{\varepsilon}$, where

$$
\begin{aligned}
& F^{*}(\varphi)(\xi)=\int_{-\infty}^{+\infty} K(1, \xi-\zeta)\left[(1-d) \varphi(\zeta-c)+\eta \int_{-\infty}^{+\infty} b^{*}(\varphi(\zeta-y-c r)) K_{D_{i}}(r-1, y) d y\right] d \zeta, \\
& F_{\varepsilon}(\varphi)(\xi)=\int_{-\infty}^{+\infty} K(1, \xi-\zeta)\left[(1-d) \varphi(\zeta-c)+\eta \int_{-\infty}^{+\infty} b_{\varepsilon}(\varphi(\zeta-y-c r)) K_{D_{i}}(r-1, y) d y\right] d \zeta .
\end{aligned}
$$

By Lemma 6 , it is easily to verify that $b^{*}$ and $b_{\varepsilon}$ satisfy all the assumptions (H1)-(H4). Therefore, the results below follow from Theorem 2 .

Lemma 7. Assume that (H1)-(H3) hold. Then for each $c>c^{*}$, there exist traveling wavefronts $\varphi^{*}(x+c n)$ and $\varphi_{\varepsilon}(x+c n)$ of (30) and (31), respectively, which satisfy $\varphi^{*}(+\infty)=W^{*}$, $\varphi^{*}(-\infty)=0$ and $\varphi_{\varepsilon}(+\infty)=W_{*}-\varepsilon, \varphi_{\varepsilon}(-\infty)=0$.

In the sequel, we will always assume $c>c^{*}$. Therefore, there exist two positive roots $\lambda_{1}(c)$ and $\lambda_{2}(c)$ with $0<\lambda_{1}(c)<\lambda_{2}(c)<\min \left\{\frac{1}{\sqrt{D_{i}}}, \frac{1}{\sqrt{D}}\right\}$. In order to proceed further, we also need the following assumptions.

Hypothesis 5 (H5). $b(w) \eta<d\left(2 w^{*}-w\right), w \in\left[W_{*}, w^{*}\right), b(w) \eta>d\left(2 w^{*}-w\right), w \in\left(w^{*}, W^{*}\right]$.

Let us first define

$$
C_{\left[0, W^{*}\right]}=\left\{\varphi \in C(\mathbb{R}, \mathbb{R}): 0 \leq \varphi(\xi) \leq W^{*}, \xi \in \mathbb{R}\right\},
$$

and for given $\lambda \in\left(0, \lambda_{1}(c)\right)$, denote

$$
X_{\lambda}=\left\{\varphi \in C(\mathbb{R}, \mathbb{R}): \sup _{\xi \in \mathbb{R}}|\varphi(\xi)| e^{-\lambda \xi}<\infty\right\},
$$




$$
\|\varphi\|_{\lambda}=\sup _{\xi \in \mathbb{R}}|\varphi(\xi)| e^{-\lambda \xi} .
$$

Clearly, $\left(X_{\lambda},\|\cdot\|_{\lambda}\right)$ is a Banach space. Then, define

$$
H^{*}(\varphi)(\xi)=(1-d) \varphi(\xi-c)+\eta \int_{-\infty}^{+\infty} K_{D_{i}}(r-1, y) b^{*}(\varphi(\xi-y-c r)) d y, \xi \in \mathbb{R},
$$

and

$$
H_{\varepsilon}(\varphi)(\xi)=(1-d) \varphi(\xi-c)+\eta \int_{-\infty}^{+\infty} K_{D_{i}}(r-1, y) b_{\varepsilon}(\varphi(\xi-y-c r)) d y, \xi \in \mathbb{R} .
$$

For any $\varphi, \psi \in\left(\mathbb{R},\left[0, W^{*}\right]\right)$ with $\varphi(\xi) \geq \psi(\xi), \xi \in \mathbb{R}$, we have

$$
H^{*}(\varphi)(\xi) \geq H^{*}(\psi)(\xi) \text { and } H_{\mathcal{\varepsilon}}(\varphi)(\xi) \geq H_{\mathcal{E}}(\psi)(\xi) \text { for all } \xi \in \mathbb{R} \text {. }
$$

Set

$$
\Gamma^{*}=\left\{\begin{array}{l|l}
\varphi \in C\left(\mathbb{R},\left[0, W^{*}\right]\right) & \begin{array}{l}
(\mathrm{i}) \varphi_{\epsilon}(\xi) \leq \varphi(\xi) \leq \varphi^{*}(\xi) \text { for all } \xi \in \mathbb{R} \\
\text { (ii) }\left|\varphi\left(\xi_{1}\right)-\varphi\left(\xi_{2}\right)\right| \leq \frac{2 W^{*}}{\sqrt{D}}\left|\xi_{1}-\xi_{2}\right| \text { for all } \xi_{1}, \xi_{2} \in \mathbb{R}
\end{array}
\end{array}\right\}
$$

Lemma 8. $\Gamma^{*} \subset X_{\lambda}$ is nonempty, convex and compact in $X_{\lambda}$.

Proof. Firstly, we note that $\varphi^{*}(\xi)$ and $\varphi_{\varepsilon}(\xi)$ are traveling wavefronts of (32) and (33), respectively, and they are obtained by iteration procedures. Set

$$
\bar{\phi}^{*}(\xi)=\min \left\{W^{*}, W^{*} e^{\lambda_{1}(c) \xi}\right\}, \bar{\phi}_{\varepsilon}(\xi)=\min \left\{W_{*}-\varepsilon,\left(W_{*}-\varepsilon\right) e^{\lambda_{1}(c) \xi}\right\} .
$$

From the proof of Theorem 2, $\bar{\phi}^{*}(\xi)$ and $\bar{\phi}_{\varepsilon}(\xi)$ are upper solutions to (34) and (35), respectively. Let

$$
\begin{aligned}
& \phi_{0}(\xi)=\bar{\phi}^{*}(\xi), \phi_{n+1}(\xi)=F^{*}\left(\phi_{n}\right)(\xi), n=0,1, \cdots, \\
& \psi_{0}(\xi)=\bar{\phi}_{\varepsilon}(\xi), \psi_{n+1}(\xi)=F_{\varepsilon}\left(\psi_{n}\right)(\xi), n=0,1, \cdots .
\end{aligned}
$$

Since $\phi_{0}(\xi) \geq \psi_{0}(\xi), b^{*}(\xi) \geq b_{\varepsilon}(\xi)$, by the monotonicity of $b^{*}$ and $b_{\varepsilon}$ on $\left[0, W^{*}\right]$, we have

$$
\phi_{1}(\xi)=F^{*}\left(\phi_{0}\right)(\xi) \geq F^{*}\left(\psi_{0}\right)(\xi) \geq F_{\varepsilon}\left(\psi_{0}\right)(\xi)=\psi_{1}(\xi),
$$

and inductively,

$$
\phi_{n}(\xi) \geq \psi_{n}(\xi), n=1,2, \cdots
$$

Moreover,

$$
\begin{aligned}
& \phi_{0}(\xi) \geq \phi_{1}(\xi) \cdots \geq \phi_{n}(\xi), \psi_{0}(\xi) \geq \psi_{1}(\xi) \cdots \geq \psi_{n}(\xi), \\
& \phi_{0}(\xi) \geq \varphi^{*}(\xi)=\lim _{n \rightarrow \infty} \phi_{n}(\xi) \geq \lim _{n \rightarrow \infty} \psi_{n}(\xi)=\varphi_{\varepsilon}(\xi) \geq 0 .
\end{aligned}
$$

Since $\phi_{0}(\xi) \in X_{\lambda}$ for $\lambda \in\left(0, \lambda_{1}(c)\right)$, we obtain $\varphi_{\varepsilon} \in X_{\lambda}$ and $\varphi^{*} \in X_{\lambda}$. Hence, $\Gamma^{*} \subset X_{\lambda}$. To prove $\Gamma^{*}$ is non-empty, it suffices to show that $\varphi^{*} \in \Gamma^{*}$.

Note that

$$
\begin{gathered}
\varphi^{*}(\xi)=F^{*}\left(\varphi^{*}\right)(\xi)=\int_{-\infty}^{+\infty} K(1, \xi-\zeta) H^{*}\left(\varphi^{*}(\zeta)\right) d \zeta, \forall \xi \in \mathbb{R}, \\
\left|H^{*}(\varphi(\xi))\right| \leq(1-d)\left|\varphi^{*}(\xi-c)\right|+\eta \int_{-\infty}^{+\infty} K_{D_{i}}(r-1, y)\left|b^{*}\left(\varphi^{*}(\xi-y-c r)\right)\right| d y \\
\leq(1-d) W^{*}+\eta b\left(W^{*}\right) \int_{-\infty}^{+\infty} K_{D_{i}}(r-1, y) d y=W^{*} .
\end{gathered}
$$


For any $\xi_{1}, \xi_{2} \in \mathbb{R}, \xi_{1}<\xi_{2}$

$$
\begin{aligned}
& \left|\varphi^{*}\left(\xi_{1}\right)-\varphi^{*}\left(\xi_{2}\right)\right|=\left|F^{*}\left(\varphi^{*}\right)\left(\xi_{1}\right)-F^{*}\left(\varphi^{*}\right)\left(\xi_{2}\right)\right| \\
\leq & \int_{-\infty}^{+\infty}\left|K\left(1, \xi_{1}-\zeta\right)-K\left(1, \xi_{2}-\zeta\right)\right|\left|H^{*}\left(\varphi^{*}(\zeta)\right)\right| d \zeta, \\
\leq & W^{*} \int_{-\infty}^{+\infty}\left|K\left(1, \xi_{1}-\zeta\right)-K\left(1, \xi_{2}-\zeta\right)\right| d \zeta, \\
\leq & \frac{W^{*}}{2 \sqrt{D}} \int_{-\infty}^{\xi_{1}}\left|e^{-\frac{1}{\sqrt{D}}\left(\xi_{1}-\zeta\right)}-e^{-\frac{1}{\sqrt{D}}\left(\xi_{2}-\zeta\right)}\right| d \zeta \\
+ & \frac{W^{*}}{2 \sqrt{D}} \int_{\xi_{2}}^{+\infty}\left|e^{\frac{1}{\sqrt{D}}\left(\xi_{1}-\zeta\right)}-e^{\frac{1}{\sqrt{D}}\left(\xi_{2}-\zeta\right)}\right| d \zeta \\
+ & \frac{W^{*}}{2 \sqrt{D}} \int_{\xi_{1}}^{\xi_{2}} e^{-\frac{1}{\sqrt{D}}\left(\xi_{2}-\zeta\right)} d \zeta+\frac{W^{*}}{2 \sqrt{D}} \int_{\xi_{1}}^{\xi_{2}} e^{\frac{1}{\sqrt{D}}\left(\xi_{1}-\zeta\right)} d \zeta \\
\leq & 2 W^{*}\left|1-e^{-\frac{1}{\sqrt{D}}\left(\xi_{2}-\xi_{1}\right)}\right| \leq \frac{2 W^{*}}{\sqrt{D}}\left|\xi_{2}-\xi_{1}\right| .
\end{aligned}
$$

Therefore, $\varphi^{*} \in \Gamma^{*}$. Similarly, we can prove $\varphi_{\varepsilon} \in \Gamma^{*}$. Next, we proof that $\Gamma^{*}$ is convex in $X_{\lambda}$. For any $\varphi, \psi \in \Gamma, 0 \leq \lambda \leq 1$,

$$
\lambda \varphi_{\varepsilon}(\xi) \leq \lambda \varphi(\xi) \leq \lambda \varphi^{*}(\xi), \quad(1-\lambda) \psi_{\varepsilon}(\xi) \leq(1-\lambda) \psi(\xi) \leq(1-\lambda) \psi^{*}(\xi) .
$$

Thus, we have

$$
\begin{gathered}
\lambda \varphi_{\varepsilon}(\xi)+(1-\lambda) \psi_{\varepsilon}(\xi) \leq \lambda \varphi(\xi)+(1-\lambda) \psi(\xi) \leq \lambda \varphi^{*}(\xi)+(1-\lambda) \psi^{*}(\xi), \\
\left|\lambda \varphi\left(\xi_{1}\right)+(1-\lambda) \psi\left(\xi_{1}\right)-\left[\lambda \varphi\left(\xi_{2}\right)+(1-\lambda) \psi\left(\xi_{2}\right)\right]\right| \leq \frac{2 W^{*}}{\sqrt{D}}\left|\xi_{2}-\xi_{1}\right| .
\end{gathered}
$$

Therefore, $\lambda \varphi+(1-\lambda) \psi \in \Gamma^{*}$. Proofs of the other conclusions of Lemma 8 are easy, we omit them. This complete the proof.

Now, we are in position to state our main result.

Theorem 3. Assume that (H1)-(H3) hold true. Then, there exists $c_{*}>0$, such that for every $c>c^{*},(14)$ admits a traveling wave solution $\varphi(x+c n)$ satisfying

$$
\lim _{\xi \rightarrow-\infty} \varphi(\xi)=0, W_{*} \leq \liminf _{\xi \rightarrow+\infty} \varphi(\xi) \leq \limsup _{\xi \rightarrow+\infty} \varphi(\xi) \leq W^{*} .
$$

Furthermore, if (H5) hold, then $\varphi$ connects the equilibrium 0 and $w^{*}$, i.e., $\lim _{\xi \rightarrow-\infty} \varphi(\xi)=0$, $\lim _{\xi \rightarrow+\infty} \varphi(\xi)=w^{*}$.

Proof. We will use Schauder fixed point theorem to prove that (14) has a traveling wave solution.

Recall that

$$
\mathbf{F}(\varphi)(\xi)=\int_{-\infty}^{+\infty} K(1, \xi-\zeta) H(\varphi)(\zeta) d \zeta
$$

where

$$
H(\varphi)(\xi)=(1-d) \varphi(\xi-c)+\eta \int_{-\infty}^{+\infty} K_{D_{i}}(r-1, y) b(\varphi(\xi-y-c r)) d y, \xi \in \mathbb{R} .
$$

Obviously, for any $\varphi \in \Gamma^{*}$,

$$
0 \leq H_{\mathcal{\varepsilon}}(\varphi)(\xi) \leq H(\varphi)(\xi) \leq H^{*}(\varphi)(\xi) \leq W^{*}, \forall \xi \in \mathbb{R} .
$$


It follows that

$$
0 \leq \mathbf{F}(\varphi)(\xi) \leq W^{*},
$$

and, hence, $\mathbf{F}: \Gamma^{*} \rightarrow C\left(\mathbb{R},\left[0, W^{*}\right]\right)$ is well defined.

For any $\varphi, \psi \in X_{\lambda}$, by the continuity of $b^{\prime}(w)$ on $\left[0, W^{*}\right]$, we have

$$
\begin{aligned}
|H(\varphi)(\xi)-H(\psi)(\xi)| e^{-\lambda \xi} \leq & (1-d)|\varphi(\xi-c)-\psi(\xi-c)| e^{-\lambda \xi} \\
& +\eta \int_{-\infty}^{+\infty} K_{D_{i}}(r-1, y)|b(\varphi(\xi-y-c r))-b(\psi(\xi-y-c r))| e^{-\lambda \xi} d y \\
\leq & (1-d)\|\varphi-\psi\|_{\lambda}+g^{\prime}(0) \eta e^{-c r \lambda} \int_{-\infty}^{+\infty} K_{D_{i}}(r-1, y) e^{-\lambda y}\|\varphi-\psi\|_{\lambda} d y \\
\leq & \frac{(1-d)+g^{\prime}(0) \eta e^{-c r \lambda}}{\left(1-D_{i} \lambda^{2}\right)^{r-1}}\|\varphi-\psi\|_{\lambda} \\
= & M\|\varphi-\psi\|_{\lambda},
\end{aligned}
$$

where $M=\frac{(1-d)+g^{\prime}(0) \eta e^{-c r \lambda}}{\left(1-D_{i} \lambda^{2}\right)^{r-1}}$. Therefore, we have

$$
\begin{aligned}
|\mathbf{F}(\varphi)(\xi)-\mathbf{F}(\psi)(\xi)| e^{-\lambda \xi} & \leq \int_{-\infty}^{+\infty} K(1, \xi-\zeta)|H(\varphi)(\zeta)-H(\psi)(\zeta)| e^{-\lambda \xi} d \zeta \\
& =\int_{-\infty}^{+\infty} K(1, \xi-\zeta) e^{-\lambda(\xi-\zeta)}|H(\varphi)(\zeta)-H(\psi)(\zeta)| e^{-\lambda \zeta} d \zeta \\
& \leq \frac{M}{1-\lambda^{2} D}\|\varphi-\psi\|_{\lambda},
\end{aligned}
$$

which yields

$$
\|\mathbf{F}(\varphi)(\xi)-\mathbf{F}(\psi)(\xi)\|_{\lambda} \leq M_{0}\|\varphi-\psi\|_{\lambda} .
$$

where $M_{0}=\frac{M}{1-\lambda^{2} D}$. Therefore, $\mathbf{F}\left(\Gamma^{*}\right) \subseteq C\left(\mathbf{R},\left[0, W^{*}\right]\right)$ is continuous.

In what follows, we verify that $\mathbf{F}\left(\Gamma^{*}\right) \subseteq \Gamma^{*}$. Note that $\varphi_{\varepsilon}(\xi)$ is a solution of (35), i.e.,

$$
\varphi_{\epsilon}(\xi)=\int_{-\infty}^{+\infty} K(1, \xi-\zeta) H_{\epsilon}\left(\varphi_{\varepsilon}\right)(\zeta) d \zeta
$$

Then, for any $\varphi \in \Gamma^{*}$,

$$
\begin{aligned}
\mathbf{F}(\varphi)(\xi) & =\int_{-\infty}^{+\infty} K(1, \xi-\zeta) H(\varphi)(\zeta) d \zeta \\
& \geq \int_{-\infty}^{+\infty} K(1, \xi-\zeta) H_{\varepsilon}(\varphi)(\zeta) d \zeta \\
& \geq \int_{-\infty}^{+\infty} K(1, \xi-\zeta) H_{\varepsilon}\left(\varphi_{\varepsilon}\right)(\zeta) d \zeta \\
& =\varphi_{\varepsilon}(\xi)
\end{aligned}
$$

Similarly, $\mathbf{F}(\varphi)(\xi) \leq \varphi^{*}(\xi)$ for all $\xi \in \mathbb{R}$.

For any $\varphi \in \Gamma^{*}$ and $\xi_{1}, \xi_{2} \in \mathbf{R}$ with $\xi_{1}<\xi_{2}$, by the same argument as in the proof Lemma 8, we have

$$
\left|\mathbf{F}(\varphi)\left(\xi_{1}\right)-\mathbf{F}(\varphi)\left(\xi_{2}\right)\right| \leq \frac{2 W^{*}}{\sqrt{D}}\left|\xi_{1}-\xi_{2}\right| .
$$

Therefore, we conclude that $\mathbf{F}(\varphi) \in \Gamma^{*}$ for all $\varphi \in \Gamma^{*}$.

Using Schauder's fixed point theorem, we obtain that $\mathbf{F}$ has a fixed point $\varphi$ in $\Gamma^{*} \subset X_{\lambda}$, which satisfies

$$
\varphi(\xi)=\int_{-\infty}^{+\infty} K(1, \xi-\zeta)\left[(1-d) \varphi(\zeta-c)+\eta \int_{-\infty}^{+\infty} K_{D_{i}}(r-1, y) b(\varphi(\zeta-y-c r)) d y\right] d \zeta .
$$


and

$$
\varphi_{\epsilon}(\xi) \leq \varphi(\xi) \leq \varphi^{*}(\xi) \text { for all } \xi \in \mathbb{R} \text {. }
$$

Taking limits $\xi \rightarrow-\infty$ and $\xi \rightarrow+\infty$ in (43), respectively, we have $\varphi(-\infty)=0$ and

$$
W_{*}-\varepsilon \leq \liminf _{\xi \rightarrow+\infty} \varphi(\xi) \leq \limsup _{\xi \rightarrow+\infty} \varphi(\xi) \leq W^{*} .
$$

Then, letting $\varepsilon \rightarrow 0^{+}$in the above inequality, we obtain

$$
W_{*} \leq \liminf _{\xi \rightarrow+\infty} \varphi(\xi) \leq \limsup _{\xi \rightarrow+\infty} \varphi(\xi) \leq W^{*}
$$

This finishes the first part of the proof of Theorem 3.

Next, we denote

$$
\alpha=\liminf _{\xi \rightarrow+\infty} \varphi(\xi), \beta=\limsup _{\xi \rightarrow+\infty} \varphi(\xi)
$$

Then $W_{*} \leq \alpha \leq \beta \leq W^{*}$. We will verify that $\alpha=\beta$.

If this is invalid, then $\alpha<\beta$. It is easy to see that if there exists a large number $M>0$, such that $\varphi^{\prime} \geq 0$ or $\varphi^{\prime} \leq 0$ on $[M,+\infty)$, then $\lim _{\xi \rightarrow+\infty} \varphi(\xi)$ exists and $\alpha=\beta$, which leads to a contradiction. So there must be a sequence $\left\{\xi_{j}\right\}_{j \in \mathbb{N}}$ with $\xi_{j} \rightarrow+\infty$ as $j \rightarrow+\infty$, such that $\varphi^{\prime}\left(\xi_{j}\right)=0, \varphi^{\prime \prime}\left(\xi_{j}\right) \leq 0$ and $\varphi\left(\xi_{j}\right) \rightarrow \beta$ as $j \rightarrow+\infty$. It follows from (14) that

$$
\begin{aligned}
0 & =D \varphi^{\prime \prime}\left(\xi_{j}\right)-\varphi\left(\xi_{j}\right)+(1-d) \varphi\left(\xi_{j}-c\right)+\eta \int_{-\infty}^{+\infty} K_{D_{i}}(r-1, y) b\left(\varphi\left(\xi_{j}-y-c r\right)\right) d y \\
& \leq-\varphi\left(\xi_{j}\right)+(1-d) \varphi\left(\xi_{j}-c\right)+\eta \int_{-\infty}^{+\infty} K_{D_{i}}(r-1, y) b\left(\varphi\left(\xi_{j}-y-c r\right)\right) d y,
\end{aligned}
$$

thus

$$
\varphi\left(\xi_{j}\right)-(1-d) \varphi\left(\xi_{j}-c\right) \leq \eta \int_{-\infty}^{+\infty} K_{D_{i}}(r-1, y) b\left(\varphi\left(\xi_{j}-y-c r\right)\right) d y
$$

For any $\varepsilon>0$, there exists a sufficiently large constant $N>0$, such that

$$
d W^{*} / \eta \int_{|y|>N} K_{D_{i}}(r-1, y) d y<\frac{\varepsilon}{2} .
$$

Due to the continuity of $b$, we can choose $\delta>0$ with $\delta<\varepsilon$ satisfying

$$
\max \{b(w) \mid w \in[\alpha-\delta, \beta+\delta]\}<\max \{b(w) \mid \alpha \leq w \leq \beta\}+\frac{\varepsilon}{2 \eta} .
$$

For such $\delta>0$, take $N_{1}>0$, such that

$$
\varphi(\xi) \in[\alpha-\delta, \beta+\delta], \text { for all } \xi \geq N_{1}
$$

Then choose $J_{0}>0$, such that

$$
\xi_{j} \geq N_{1}+N+c r \text {, for all } j \geq J_{0} .
$$

Therefore, for $j \geq J_{0}$, we have 


$$
\begin{aligned}
\varphi\left(\xi_{j}\right)-(1-d) \varphi\left(\xi_{j}-c\right) & \leq \eta \int_{|y| \leq N} K_{D_{i}}(r-1, y) b\left(\varphi\left(\xi_{j}-y-c r\right)\right) d y \\
& +\eta \int_{|y|>N} K_{D_{i}}(r-1, y) b\left(\varphi\left(\xi_{j}-y-c r\right)\right) d y \\
& \leq \eta \max \{b(w) \mid w \in[\alpha-\delta, \beta+\delta]\}+d W^{*} / \eta \int_{|y|>N} K_{D_{i}}(r-1, y) d y \\
& \leq \eta \max \{b(w) \mid \alpha \leq w \leq \beta\}+\varepsilon .
\end{aligned}
$$

Let $j \rightarrow+\infty$, we find

$$
d \beta \leq \eta \max \{b(w) \mid \alpha \leq w \leq \beta\}+\varepsilon,
$$

It follows from $\varepsilon \rightarrow 0^{+}$that

$$
d \beta \leq \eta \max \{b(w) \mid \alpha \leq w \leq \beta\} .
$$

Similarly, we have

$$
d \alpha \geq \eta \min \{b(w) \mid \alpha \leq w \leq \beta\} .
$$

If $\alpha<\beta \leq w^{*}$, then by (47), we have $d \alpha \geq \eta \min \{b(w) \mid \alpha \leq w \leq \beta\}>d \alpha$, a contradiction. If $w^{*} \leq \alpha<\beta$, then (46) implies that $d \beta \leq \eta \max \{b(w) \mid \alpha \leq w \leq \beta\}<d \beta$, also a contradiction. Therefore, we must have $\alpha<w^{*}<\beta$.

Let $w_{1}, w_{2} \in[\alpha, \beta]$, such that $b\left(w_{1}\right)=\max \{b(w) \mid \alpha \leq w \leq \beta\}$ and $b\left(w_{2}\right)=\min \{b(w) \mid \alpha \leq w \leq \beta\}$. We have the following three cases:

Case (i). $w^{*} \leq w_{1} \leq \beta$. If $w_{1}=\beta$, then $d \beta \leq \eta b(\beta)$ follows from (46), which is impossible since $\beta>w^{*}$. Therefore, we have $w_{1}<\beta$ and, hence,

$$
d \beta \leq \eta \max \{b(w) \mid \alpha \leq w \leq \beta\}=b\left(w_{1}\right) \eta \leq d w_{1}<d \beta,
$$

which is a contradiction;

Case (ii). $\alpha \leq w_{2} \leq w^{*}$. Using the similar argument that used in (i), we find $\alpha<w_{2}$ and

$$
d \alpha \geq \eta \min \{b(w) \mid \alpha \leq w \leq \beta\}=b\left(w_{2}\right) \eta \geq d w_{2}>d \alpha,
$$

which is also a contradiction;

Case (iii). $w_{1}<w^{*}<w_{2}$. In this case, we have $w_{1}=\alpha$ and $w_{2}=\beta$. Otherwise, we have

$$
\begin{aligned}
d \beta-d \alpha & \leq \eta b\left(w_{1}\right)-\eta b\left(w_{2}\right) \\
& <d\left(2 w^{*}-w_{1}\right)-d\left(2 w^{*}-w_{2}\right)=d\left(w_{2}-w_{1}\right) \\
& <d \beta-d \alpha
\end{aligned}
$$

which is impossible.

Thus, $\alpha=\beta$, and hence $\lim _{\xi \rightarrow+\infty} \varphi(\xi)=\alpha \in\left[W_{*}, W^{*}\right]$ exists. Using the Lebesgue's dominated convergence theorem and taking the limit as $\xi \rightarrow+\infty$ in (42), we have

$$
\begin{aligned}
\alpha & =\lim _{\zeta \rightarrow+\infty} \int_{-\infty}^{+\infty} K(1, \xi-\zeta)\left[(1-d) \varphi(\zeta-c)+\eta \int_{-\infty}^{+\infty} K_{D_{i}}(r-1, y) b(\varphi(\zeta-y-c r)) d y\right] d \zeta \\
& =(1-d) \alpha+\eta b(\alpha)
\end{aligned}
$$

which yields $d \alpha=\eta b(\alpha)$, hence $\lim _{\xi \rightarrow+\infty} \varphi(\xi)=\alpha=w^{*}$. 


\section{Discussion}

In this paper, we studied the traveling wave solutions of the temporally discrete reaction-diffusion equation with monotone and non-monotone birth functions, respectively. Now we compare our results with the counterparts for continuous model. In [2], the authors studied the existence of traveling wavefronts of following equation,

$$
\frac{\partial w}{\partial t}=D \frac{\partial^{2} w}{\partial x^{2}}-d w+\varepsilon p \int_{-\infty}^{+\infty} b(w(t-r, y)) f_{\alpha}(x-y) d y
$$

where they took a particular birth function $b(w)=p w e^{-a w}, p>0$ and $a>0$ are parameters. They showed that if $1<\frac{\varepsilon p}{d} \leq e$, then there exists $\bar{c}^{*}>0$ such that for every $c>\bar{c}^{*}$, (48) admits a traveling wavefront solution connecting the trivial equilibrium $w_{1}=0$ and positive equilibrium $w_{2}=\frac{1}{a} \ln \frac{\varepsilon p}{d}$. In [23], the author established a general existence result of traveling wave solutions for non-local reaction diffusion equation. As a special case, he proved that if $e<\frac{\varepsilon p}{d} \leq e^{2}$, (48) still possess a traveling wave solution connecting $w_{1}$ and $w_{2}$.

Using the non-standard discretization as the form of (1), the corresponding temporally discrete reaction diffusion equation reads as

$$
\begin{aligned}
w(n+1, x)= & D \Delta w(n+1, x)+(1-d) w(n, x) \\
& +\varepsilon p \int_{-\infty}^{+\infty} K_{D_{i}}(r-1, x-y) w(n+1-r, y) e^{-a w(n+1-r, y)} d y .
\end{aligned}
$$

If $1<\frac{\varepsilon p}{d} \leq e$, then the assumptions (H1)-(H4) can be easily verified. By Theorem 2, there exists $c^{*}>0$, such that, for every $c>c^{*},(49)$ admits a traveling wavefront with speed c. When $e<\frac{\varepsilon p}{d} \leq e^{2}$, by Theorem 3, we can prove that (49) still possess a traveling wave solution connecting $w_{1}$ and $w_{2}$. This implies that in the sense of the existence of traveling wavefronts, (49) is a dynamically persistent discretization.

Now that both (48) and (49) have critical propagation speeds $\bar{c}^{*}$ and $c^{*}$, respectively. What is the relationship between the two propagation speeds $\bar{c}^{*}$ and $c^{*}$ ? To answer this question, we can consider following two functions,

$$
\begin{aligned}
& \bar{\Delta}(c, \lambda)=D \lambda^{2}-d-c \lambda+\varepsilon p e^{\alpha \lambda^{2}-\lambda c r}, \\
& \Delta(c, \lambda)=D \lambda^{2}-1+(1-d) e^{-\lambda c}+\frac{\varepsilon p e^{-\lambda c r}}{\left(1-D_{i} \lambda^{2}\right)^{r-1}} .
\end{aligned}
$$

where $\alpha=(r-1) D_{i}$. In fact, $\bar{\Delta}(c, \lambda)=0$ and $\Delta(c, \lambda)=0$ are characteristic equations of linearizations at zero of wave profile equations corresponding to (48) and (49).

By direct computations, we find

$$
-d-c \lambda<-1+(1-d) e^{-\lambda c}, e^{\alpha \lambda^{2}}<\frac{1}{\left(1-D_{i} \lambda^{2}\right)^{r-1}}, \forall \lambda \in\left(0, \frac{1}{\sqrt{D_{i}}}\right),
$$

which implies that for any given $c>0, \Delta(c, \lambda)>\bar{\Delta}(c, \lambda)$ for $\lambda \in\left(0, \frac{1}{\sqrt{D_{i}}}\right)$.

By the definition of $\bar{c}^{*}$, there exists a unique $\lambda=\lambda\left(\bar{c}^{*}\right)>0$, such that $\bar{\Delta}\left(\bar{c}^{*}, \lambda\left(\bar{c}^{*}\right)\right)=0$. Moreover, $\bar{\Delta}\left(\bar{c}^{*}, \lambda\right)>0$ for $0<\lambda \neq \lambda\left(\bar{c}^{*}\right)$. Consequently, $\Delta\left(\bar{c}^{*}, \lambda\right)>\bar{\Delta}\left(\bar{c}^{*}, \lambda\right) \geq 0$ for any $0 \leq \lambda \leq \frac{1}{\sqrt{D_{i}}}$. It follows that $c^{*}>\bar{c}^{*}$.

Author Contributions: Writing—original draft preparation, H.G.; writing—review and editing, Z.G. and H.G. Both authors have read and agreed to the published version of the manuscript.

Funding: This project was supported by the National Natural Science Foundation of China (No. 11771104), Science and Technology Planning Project of Guangdong Province of China (2020A1414010106), The Natural Science Foundation of Guangdong Province of China (2021A1515010310) and Graduate Basic Innovation Project of Guangzhou University (No. 2019-GDJC-D03). 
Institutional Review Board Statement: Not applicable.

Informed Consent Statement: Not applicable.

Data Availability Statement: Not applicable.

Conflicts of Interest: The authors declare no conflict of interest.

\section{References}

1. Guo, Z.; Guo, H.; Chen, Y. Traveling wavefronts of a delayed temporally discrete reaction-diffusion equation. J. Math. Anal. Appl. 2021, 496, 124787. [CrossRef]

2. So, J.; Wu, J.; Zou, X. A reaction-diffusion model for a single species with age structure. I Travelling wavefronts on unbounded domains. Proc. R. Soc. Lond. A. 2001, 457, 1841-1853. [CrossRef]

3. Britton, N. Reaction-Diffusion Equations and Their Applications to Biology; Academic Press: New York, NY, USA, 1986.

4. Bian, Q.; Zhang, W.; Yu, Z. Temporally discrete three-species Lotka-Volterra competitive systems with time delays. Taizwan J. Math. 2016, 20, 49-75. [CrossRef]

5. Fife, P. Mathematical Aspects of Reaction and Diffusing Systems; Lecture Notes in Biomathematics; Springer: Berlin, Germany, 1979; Volume 28.

6. Freedman, H. Deterministic Mathematical Models in Population Ecology; Marcel Dekker: New York, NY, USA, 1980.

7. Goh, B. Management and Analysis of Biological Populations; Elsevier: Amsterdam, The Netherlands, 1980.

8. Martin, R.H.; Smith, H.L. Abstract functional differential equations and reaction-diffusion systems. Trans. Am. Math. Soc. 1990, $321,1-44$.

9. Murray, J.D. Mathematial Biology; Biomathematics; Springer: Berlin, Germany, 1993; Volume 19.

10. Weinberger, H.F. Long-time behavior of a class of biological models. SIAM J. Math. Anal. 1982, 13, 353-396. [CrossRef]

11. Mickens, R.E. Nonstandard Finite Difference Models of Differential Equations; World Scientific: Singapore, 1994.

12. Mickens, R.E. Dynamic consistency: A fundamental principle for constructing nonstandard finite difference dchemes for differential equations. J. Differ. Equ. Appl. 2005, 11, 645-653. [CrossRef]

13. Anguelov, R.; Dumont, Y.; Lubuma, J.M.-S.; Shillor, M. Dynamically consistent nonstandard finite difference schemes for epidemiological models. J. Comput. Appl. Math. 2014, 255, 161-182. [CrossRef]

14. Dang, Q.A.; Hoang, M.T. Dynamically consistent discrete metapopulation model. J. Differ. Equ. Appl. 2016, 22, 1325-1349. [CrossRef]

15. Dimitrov, D.T.; Kojouharov, H.V. Dynamically consistent numerical methods for general productive-destructive systems. J. Differ. Equ. Appl. 2011, 17, 1721-1736. [CrossRef]

16. Roeger, L.-I.W. Dynamically consistent discrete-time Lotka-Volterra competition models. Discrete Contin. Dyn. Syst. 2009, 2009, 650-658

17. Suryanto, A.; Kusumawinahyu, W.M.; Darti, I.; Yanti, I. Dynamically consistent discrete epidemic model with modified saturated incidence rate. Comput. Appl. Math. 2013, 32, 373-383. [CrossRef]

18. Weinberger, H.F.; Lewis, M.A.; Li, B. Analysis of linear determinacy for spread in cooperative models. J. Math. Biol. 2002, 45, 183-218. [CrossRef] [PubMed]

19. Kot, M.; Schaffer, W.M. Discrete-time growth-dispersal models. J. Math. Biol. 1986, 80, 109-136. [CrossRef]

20. Lin, G.; Li, W. Traveling wavefronts in temporally discrete reaction-diffusion equations with delay. Nonlinear Anal. Real World Appl. 2008, 9, 197-205. [CrossRef]

21. Xue, H.; Huang, J.; Yu, Z. Invasion traveling wave solutions in temporally discrete random-diffusion systems with delays. Discret. Contin. Dyn. Syst. Ser. S 2017, 10, 1107-1131. [CrossRef]

22. Xue, H.; Huang, J.; Yu, Z. Existence and asymptotic behavior of invasion wave solutions in temporally discrete diffusion systems with delays. Int. J. Biomath. 2018, 11, 1850016. [CrossRef]

23. Ma, S. Traveling waves for non-local delayed diffusion equations via auxiliary equations. J. Differ. Equ. 2007, $237,259-277$. [CrossRef]

24. Ma, S.; Weng, P.; Zou, X. Asymptotic speed of propagation and traveling wavefronts in a non-local delayed lattice differential equation. Nonlinear Anal. 2006, 65, 1858-1890. [CrossRef] 